Research Article

\title{
The Effects of Ammonium Polyacrylate and Diammonium Citrate as Base and Acid Dispersion Agents on Yttria-Stabilized Zirconia (3Y-TZP) Dispersion Properties
}

\author{
Pestaria Sinaga $^{1}$ and Sung-Hwan Bae $\mathbb{D}^{2}$ \\ ${ }^{1}$ Department of Mechatronics Engineering, Kyungnam University, Changwon, Republic of Korea \\ ${ }^{2}$ Department of Nano Science and Engineering, Kyungnam University, Changwon, Republic of Korea \\ Correspondence should be addressed to Sung-Hwan Bae; shbae@kyungnam.ac.kr
}

Received 24 October 2017; Revised 24 January 2018; Accepted 19 February 2018; Published 4 April 2018

Academic Editor: Fabrizio Pirri

Copyright (c) 2018 Pestaria Sinaga and Sung-Hwan Bae. This is an open access article distributed under the Creative Commons Attribution License, which permits unrestricted use, distribution, and reproduction in any medium, provided the original work is properly cited.

\begin{abstract}
Stable slurries in dispersions of 3Y-TZP in aqueous suspension with the addition of different concentrations of dispersants such as ammonium polyacrylate (APA) and diammonium citrate (DAC) were investigated. The dispersion properties were investigated by measuring the particle size, zeta potential, sedimentation, and viscosity as a function of the wt.\% of the dispersant. Both dispersant agents were attached to the 3Y-TZP surface by the carboxylic group, as shown by the FTIR results. The addition of dispersants was found to produce more dispersed and stabilized aqueous suspension. As shown the viscosity result, that there is no being viscosity peak has been occurring and viscosity going decrease as the shear rates increasing which mean that the suspension has shear thinning behavior and there is no agglomeration as the shear rate is increased. It was determined that $3.5 \mathrm{wt} \%$ of DAC and APA produced the best and most stable slurry; when $3.5 \mathrm{wt} . \%$ of DAC and APA was added, the zeta potential showed the largest value in the monodisperse condition. The low $\mathrm{pH}$ value of DAC has obtained the higher zeta potential value than APA, which was assumed due to low $\mathrm{pH}$ of DAC suspension. At low $\mathrm{pH}$, the adsorption of the adsorbate will occur in a flat adsorbed, while at higher $\mathrm{pH}$ the polyelectrolyte will dangle into solution, thus reducing electrostatic repulsion as it is found in the case of APA addition. In this condition, the particle size was decreased to the lower value and the slurry's stability was obtained with the lowest sedimentation height after the sedimentation test for 30 days. The sample was milled in an attrition mill at 1,000 rpm for four hours.
\end{abstract}

\section{Introduction}

YSZ ceramics have been widely used as an advanced structural material for their excellent mechanical properties [1-3]. These advanced materials are currently utilized in a wide variety number of applications such as cutting tools, heat engine parts, body implants, sensors, capacitors, and actuators, with new applications evolving continuously [4]. As mentioned previously, in many applications, it is desirable to obtain a fine grain and uniform microstructure when producing a strong and reliable structural part; cheaper and more convenient methods of forming green bodies are of great interest [5].

Typically, when a ceramic powder such as YSZ is added to several suspensions such as water, the presence of attractive van der Waals forces results in the powder particles combining to form clusters, which leads to the formation of agglomeration, called flocculation, and the creation of potential sites for areas of high stress, leading to a reduction in the final properties [6]. In colloidal processing techniques such as direct coagulation casting (DCC), gel casting, slip casting, tape casting, and centrifugal casting, it is crucial to have a slurry with good dispersion and desirable rheological behavior because the quality of dispersion controls the casting behavior and the resulting green bodies' properties [7].

Ceramic particles can be well dispersed in an aqueous solution by controlling the surface either via electrostatic, steric, or electrosteric stabilization [5]. Controlling the surface of the powder in an aqueous solution can be done by adding the dispersant agents to counterbalance the van der Waals 
force attraction by electrostatic and steric stabilization. Electrostatic stabilization can be achieved by adding charge to the particles, so it repels each other, particularly when under the influence of the $\mathrm{pH}$. Steric stabilization can be achieved by the adsorption of a thin layer of the polymer onto the particle surface that physically prevents particles from coming close enough to each other to cause agglomeration $[8,9]$.

Generally, the combination of both electrostatic and steric stabilization is termed electrosteric stabilization and can be achieved using polyelectrolytes. Polyelectrolytes are polymers that possess a charge that is present along the length of the polymer chain, as opposed to other polymers that only have a charged species at one end of the molecule [6]. Ammonium polyelectrolyte acid, a type of weak polyelectrolyte and a derivative from the carboxyl group, would be a good candidate for YSZ dispersion [10]. However, although DAC is rarely used as a dispersant agent, an investigation found that the carboxylic group-containing agent is a good dispersant for deflocculated alumina suspensions [11], and when a metal oxide is dispersed in water, the $\mathrm{pH}$ condition of the dispersant agent should be adjusted $(\mathrm{pH}<\mathrm{pH}$ isoelectric point -2 or $\mathrm{pH}>\mathrm{pH}$ isoelectric point +2) [12] as YSZ has an isoelectric point at pH 6-8 [13, 14].

Therefore, we try to use APA and DAC as base and acid dispersant agents, as their initial respective $\mathrm{pHs}$ at 8.7 and 5.4 can stabilize YSZ suspensions. Although there were several studies about the dispersion of YSZ in aqueous suspension in this study, several conditions of dispersant agent concentration were used to find the optimum conditions for YSZ dispersion in either basic or acidic condition with APA and DAC, respectively; they will be useful for the next study of the YSZ dispersion in aqueous suspensions.

\section{Materials and Methods}

2.1. Materials. Partially stabilized zirconia powder 3Y-TZP containing $3 \mathrm{~mol} \%$ yttria (Cenotec Co., Korea) was used in this study as it was received. The median size of the initial powder $(11 \mu \mathrm{m})$ was measured with a particle size analyzer (PSA, LS 230, and N4PLUS; Beckman Coulter Corporation), and the particle size distribution is shown in Figure 1.

The 49 wt.\% of $3 \mathrm{Y}-\mathrm{TZP}$ was diluted with water as a dispersion medium, and ammonium polyacrylate (APA) and diammonium citrate (DAC) (Sigma-Aldrich Co., Korea) were added as dispersant agents at various concentrations $(0.5,1.5$, $2.5,3.5,4.5$, and 5.5 wt.\%) of the solid powder. Figure 2 shows the chemical structure of ammonium polyacrylate (APA) and diammonium citrate (DAC), respectively, and the suspension was transferred to a Teflon container and milled with an attrition mill at 1,000 rpm for four hours. Zirconia balls were used as grinding media for further dispersion. Finally, the slurry was used to measure the viscosity, while the particle size, sedimentation, FTIR, and zeta potential characterization was characterized using $1 \mathrm{~mL}$ slurry, which was redissolved in $45 \mathrm{~mL}$ distilled water.

2.2. Characterization Methods. The particle size was analyzed using a particle size analyzer (PSA RS 230), and the

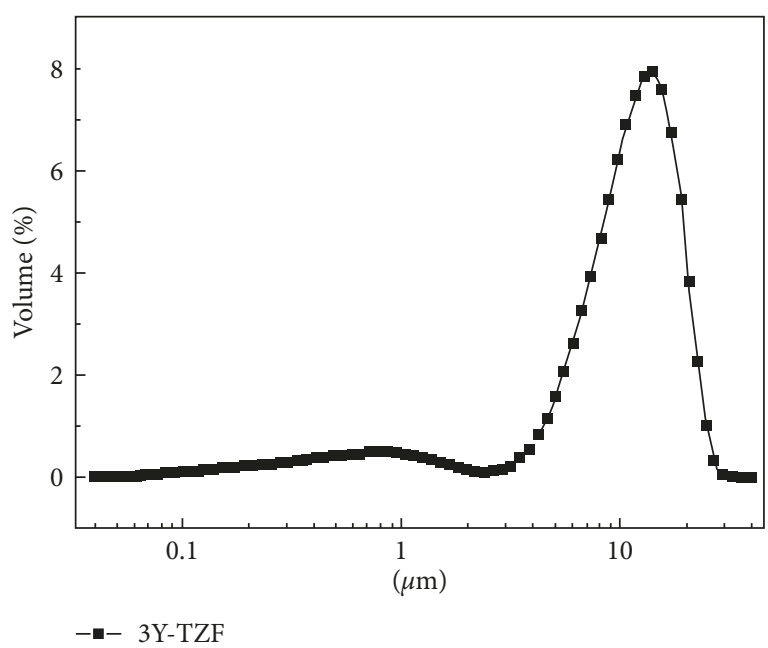

FIgURE 1: The particle size of the initial powder measured by PSA.

suspension's dispersibility was confirmed by characterizing the sedimentation. In sedimentation characterization, the sample was left to settle in a vibration-free environment; the height of the sediment formed and transparency of the supernatant formed were noted for several days. The suspension flow behavior was characterized using a viscosimeter (DV-II + PROGRAMMABLE VISCOMETER, Brookfield, USA), and the adsorption of the dispersant agent into the 3Y-TZP surface was confirmed by FTIR (FT/IR-6300, JASCO, Japan) with dilute $0.0001 \mathrm{wt} . \%$ of the slurry with distilled water.

2.2.1. Zeta Potential Measurement. After sample milling with an attrition mill for 4 hours, $0.1 \mathrm{wt}$.\% of 3Y-TZP particles in $500 \mathrm{~mL}$ of distilled water was used to determine the zeta potential. Both $\mathrm{NaOH}$ and $\mathrm{HCl}$ were used to adjust the $\mathrm{pH}$, and measurement was repeated 3 times for each measure.

2.2.2. FTIR Measurement. FTIR analysis was used to confirm the adsorption of both dispersant agents into the 3Y-TZP particle surface. The FTIR spectrum of the dispersant APA was taken in the liquid state and DAC in the solid state. For FTIR measurements, the samples used contain $3.5 \mathrm{wt} . \%$ of each dispersant agent addition. The slurry contains 1.08 wt. $\%$ of particles, which was used to determine the peak of the adsorption by using FTIR (FT/IR-6300, JASCO, Japan).

\section{Results and Discussion}

3.1. Particle Size Analysis. Figure 3 shows that the particle sizes were determined as the median size (d50) of 3Y-TZP after the addition of dispersant agents and milling for four hours. The addition of dispersant agents from 0.5 to $3.5 \mathrm{wt} . \%$ results in a reduction of particle size, and the smallest particle size occurred at the $3.5 \mathrm{wt} \%$ addition of both dispersant agents. However, when the concentration of dispersant agents increased to a value higher than $3.5 \mathrm{wt} . \%$, the particle size also increased. The increase in particle size is adjusted to the micelle formation effects when the concentration of dispersant agent addition was exceeded. 


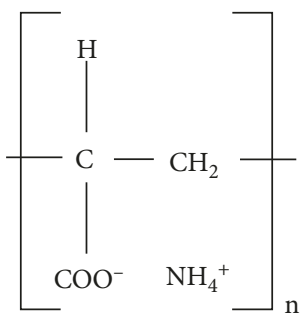

(a)

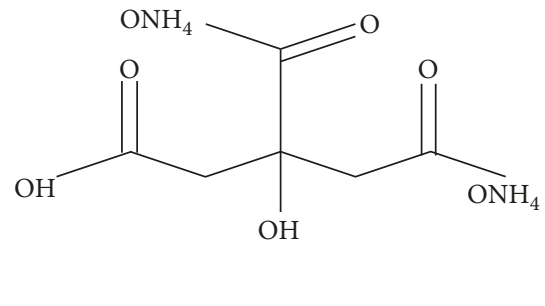

(b)

FIgURE 2: Chemical structure of both dispersants: (a) ammonium polyacrylate (APA) and (b) diammonium citrate (DAC).

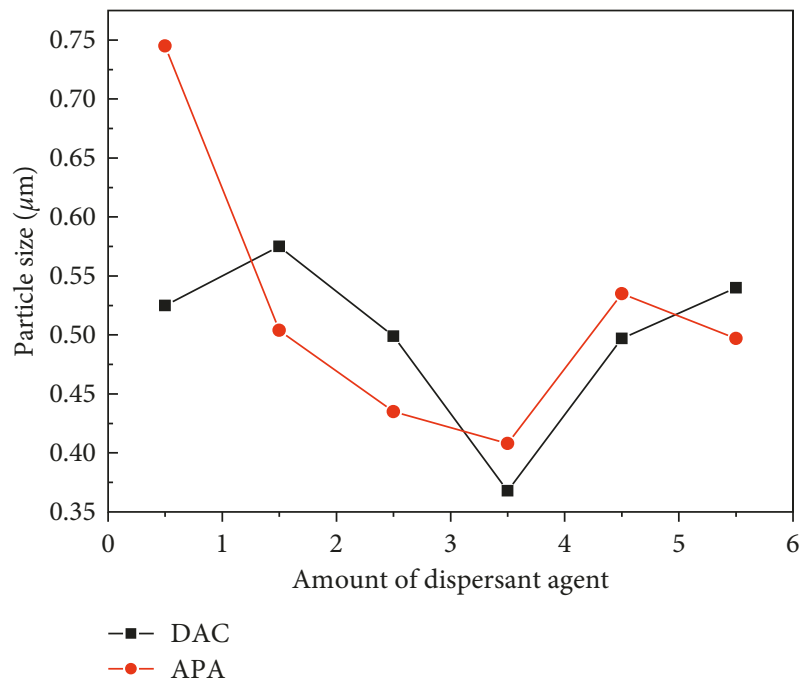

Figure 3: The particle size distribution of powder for addition of both dispersion agents.

The micelle effect, as it is shown in Figure 4, is a condition when the particle leads to reagglomeration due to the exceeded amount of dispersant agents; as the result, the particles will have flocculation. When $\mathrm{pH}$ is higher than the isoelectric point (IEP) of the suspension, under this condition, the high ionic strength in the solution will cause the adsorbate to dangle into the solution. Thus, when the particles approach the nonadsorbed dispersant agents, it will make the adsorbate that has been adsorbed onto the surface of the particles to be squeezed out of the gap and produce a local area almost identical to the pure solvent. Due to that, the particle will approach each other and build flocculation as the particle size increases, as shown for the addition of 4.5-5.5 wt.\% of dispersant agents. Nevertheless, an interesting value occurred in the addition of $5.5 \mathrm{wt} \%$ of APA. In this concentration, the particle size decreased from $0.545 \mu \mathrm{m}$ to $0.497 \mu \mathrm{m}$ with the addition of $4.5 \mathrm{wt} . \%$ and $5.5 \mathrm{wt} . \%$ of APA, respectively. The decrease in the particle size was assumed when there is further increase in the amount of APA. As the amount of nonadsorbed APA increase in the solution, they will place themselves between the clusters and thus the particle size will decrease. As it was found in the previous study, addition of high polymer concentration in nanoparticle silica will decrease the particle size and improve the suspension stability [15].
Zirconia added to the suspensions and milling at $1,000 \mathrm{rpm}$ for four hours changed the $\mathrm{pH}$ of suspensions, as shown in Table 1.

Table 1 shows that the initial $\mathrm{pH}$ of the suspension does not matter except for a high concentration of DAC added over $2.5 \mathrm{wt} . \%$ and the final $\mathrm{pH}$ value will fall to the base $\mathrm{pH}$. It was found in the previous study that addition of anionic dispersant results in the suspension's $\mathrm{pH}$ to be greater than IEP which means that the particle is negatively charged due to dispersant adsorption [16, 17]. A higher concentration of DAC addition will neutralize the $\mathrm{NH}$ from the base group, convert into a positive charge, and change the final $\mathrm{pH}$ of the suspensions into an acid condition. So it was confirmed that the addition of DAC over $2.5 \mathrm{wt}$. $\%$ will decrease the final $\mathrm{pH}$ of the slurry and create a more acidic condition. The change between $\mathrm{pH}$ of the suspension before and after milling is due to adsorption of dispersant agents into the zirconia surface. As milling time increases, the particle will become smaller, the surface area will increase, and thus adsorption will also increase. When the dispersant is added to the solution, the negatively charged carboxyl group dissociated from the dispersant which adsorbed on the positively charged $\mathrm{ZrO}_{2}$ will react with a hydroxyl group which associated with the $\mathrm{ZrO}_{2}$ particle. But because the carboxyl group adsorption is stronger than $\mathrm{OH}$, the carboxyl will attach to the zirconia surface and $\mathrm{OH}$ will be placed surrounded the particle, resulting in the increase of $\mathrm{pH}$. The smaller the particle size, the more the surface area (or more surface sites) per unit volume of the slurry, which will in general favor more hydrolysis, leading to the increase in $\mathrm{pH}$ too.

3.2. Sedimentation Behavior. The height of the sedimentation was recorded for 30 days in a vibration-free environment, as shown in Table 2. All samples have started to settle and show little tendency towards sedimentation for samples with addition of dispersant agents at concentration $3.5 \mathrm{wt} . \%$, which means that the solution is in a good dispersion condition.

\subsection{Viscosity Characterization of $3 Y-T Z P$ Slurry with} the Addition of Both Dispersant Agents (APA and DAC). Figures 5(a) and 5(b) show the rheological behavior of suspension for the addition of $0.5-5.5$ wt.\% of both APA and DAC. The addition of dispersant agents in the lowest amount of APA (0.5 wt.\%) exhibits the highest viscosity, but as the 


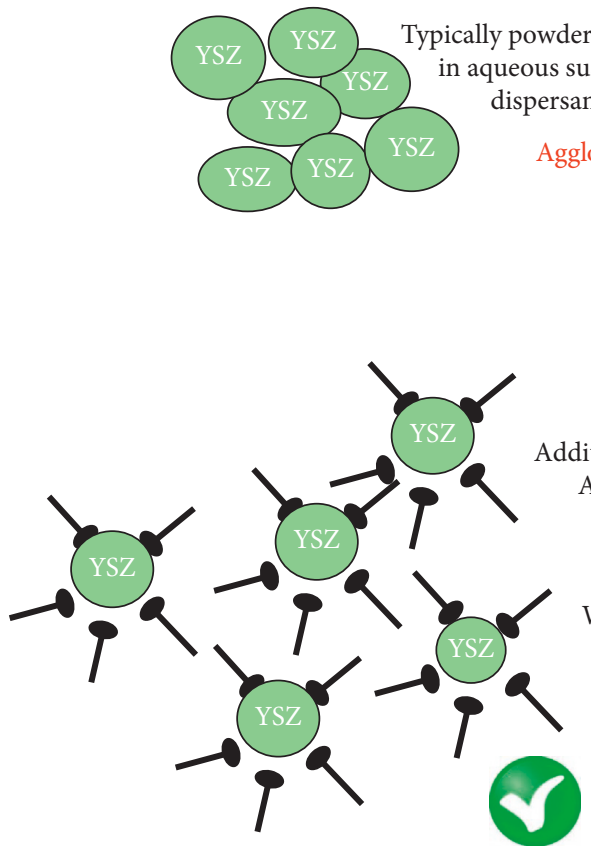

Well dispersed $\Rightarrow$ Individual particle state

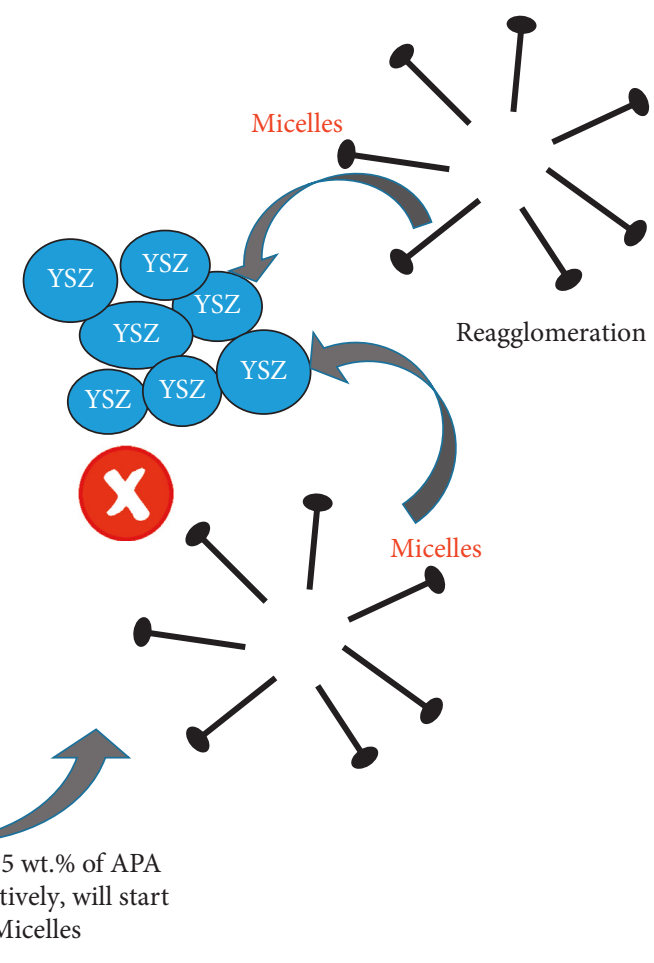

FIgURE 4: Schematic of the effect dispersant agent addition to the surface of 3Y-TZP micelle formation.

TABLE 1: The $\mathrm{pH}$ of suspensions and the slurry after milling at $1,000 \mathrm{rpm}$ for four hours.

\begin{tabular}{lcccc}
\hline $\begin{array}{l}\text { Dispersant } \\
\text { concentration (wt.\%) }\end{array}$ & $\begin{array}{c}\text { Initial pH of APA } \\
\text { suspensions }\end{array}$ & $\begin{array}{c}\text { The pH of APA suspensions } \\
\text { after milling }\end{array}$ & $\begin{array}{c}\text { Initial pH of DAC } \\
\text { suspensions }\end{array}$ & $\begin{array}{c}\text { The pH of DAC suspensions } \\
\text { after milling }\end{array}$ \\
\hline 0.5 & 8.4 & 9 & 5.34 & 9.6 \\
1.5 & 8.5 & 9.04 & 5.4 & 9.1 \\
2.5 & 8.7 & 9.06 & 5.4 & 7.8 \\
3.5 & 8.75 & 9.67 & 5.4 & 6.4 \\
4.5 & 8.84 & 9.75 & 5.4 & 6.1 \\
5.5 & 8.6 & 9.65 & 5,4 & 5.9 \\
\hline
\end{tabular}

TABle 2: Sedimentation result from 1-month testing.

\begin{tabular}{lcccccc}
\hline \multirow{2}{*}{ Dispersant agent } & \multicolumn{4}{c}{ Sedimentation result in several dispersant agent conditions (cm) } \\
& 0.5 wt.\% & 1.5 wt.\% & 2.5 wt.\% & 3.5 wt.\% & 4.5 wt.\% & 5.5 wt.\% \\
\hline DAC & 0.4 & 0.4 & 0.4 & 0.25 & 0.4 & 0.4 \\
APA & 0.45 & 0.4 & 0.4 & 0.25 & 0.4 & 0.4 \\
\hline
\end{tabular}

dispersant agents concentration increased up to $1.5 \mathrm{wt} \%$ the viscosity is decreased significantly to a lower value. A lower viscosity improves the dispersant condition; nevertheless, Figure 5(a) shows that the viscosity increases as dispersant agents are added. Measuring the viscosity can demonstrate two conditions of the dispersion state: the first state at 1.5-3.5 wt.\% and the second state at 4.5-5.5 wt.\%. At 1.5-3.5 wt.\%, it was assumed that the suspension stability was increased, and the optimum condition at $3.5 \mathrm{wt}$.\% was found as the optimum stabilized condition. Although the viscosity of $1.5 \mathrm{wt} . \%$ is lower than $3.5 \mathrm{wt} . \%$, it was not insignificantly valued. When polyelectrolytes such as APA is added in low concentration and $\mathrm{pH}$ below the dissociation point of the acid group, the electrostatic repulsion along the polymer length will make the polyelectrolyte charged and stretched. Then, it will be absorbed into the powder surface by a thin, flat train configuration with loop suppressed, which means that only electrostatic stabilization occurs. But, as the concentration of APA increases to the higher concentration, polyelectrolytes will be associated and lead to the suppression of the electrostatic repulsion, which means that suspension is stabilized by steric stabilization only. Due to this reason, we assumed 3.5 wt.\% concentration addition of APA as the optimum condition. The increase in the viscosity value was also assumed due to the decrease of the particle size $[18,19]$ as APA concentration addition from 1.5 to $3.5 \mathrm{wt}$ \%. The second state 


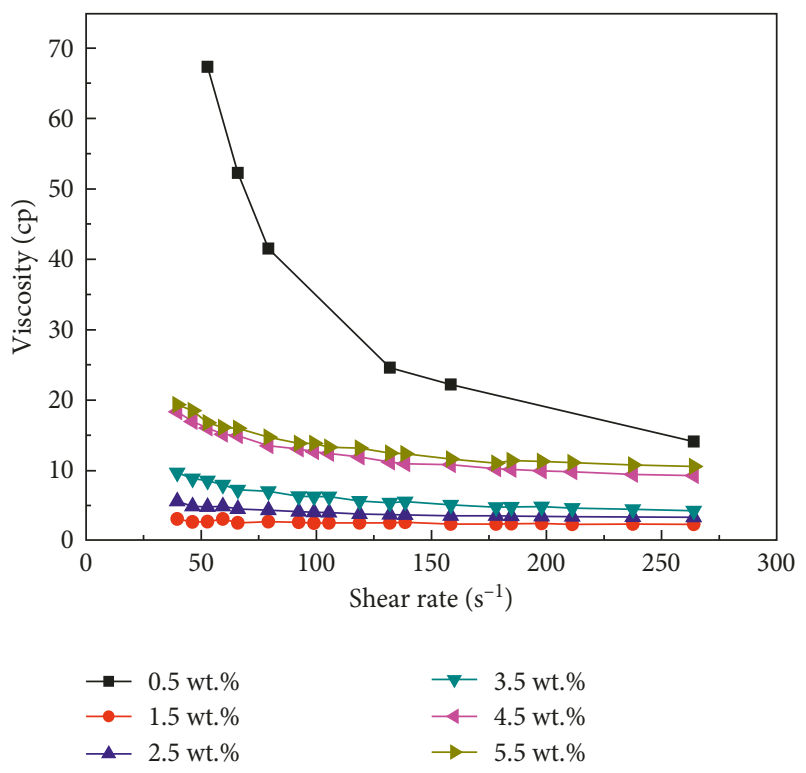

(a)

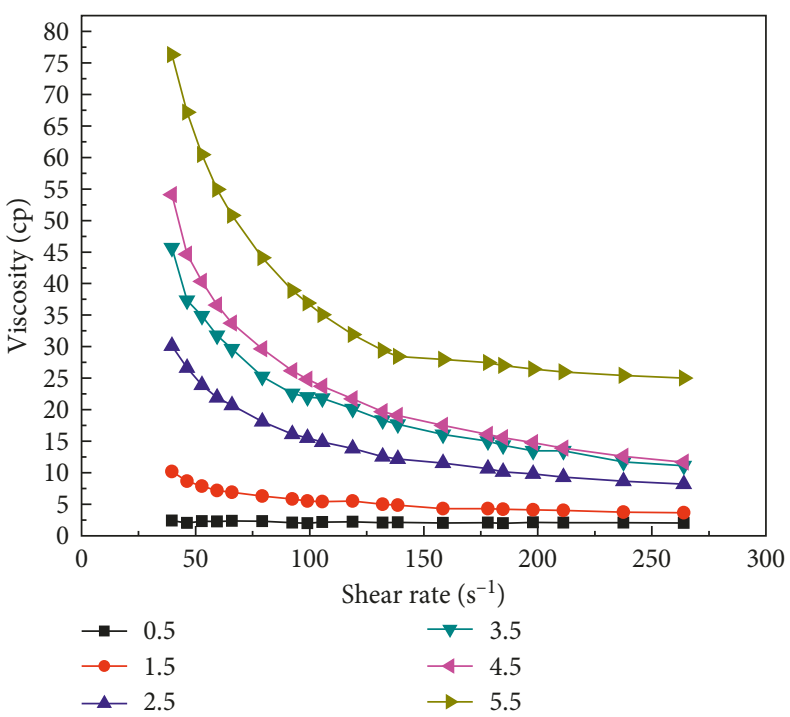

(b)

FIGURE 5: The viscosity of 3Y-TZP powder in various conditions of addition of both dispersant agents: (a) 3Y-TZP-APA and (b) 3Y-TZP-DAC.

at $4.5-5.5 \mathrm{wt} . \%$ was assumed to the unstable state because the viscosity was increasing significantly from $3.5 \mathrm{wt}$. $\%$ to $4.5 \mathrm{wt} . \%$. Once the adsorption completed, the increase of dispersant concentration will lead to particle flocculation due to the micelle effect. Due to this reason, the viscosity will increase significantly.

In Figure 5(b), the 3Y-TZP-DAC suspension exhibited shear thinning behavior, and viscosity increased when the concentration of dispersant agents increased; this was assumed due to the nonpolymeric and low molecular weight of DAC. Nonpolymeric and small molecular weight of DAC was predicted to make the particle approach easier and make the network as the concentration exceeds. Once the adsorption is completed, an increase in the amount of DAC (>3.5 wt.\%) will increase the ionic strength significantly, which will reduce the electrical double layer repulsion. The nonadsorbed carboxyl group that is generated from DAC will remain in the solution and they will build a network easier due to the small molecular weight and nonpolymeric nature of DAC. As a result, the particle will interconnect to each other and lead to flocculation, as the resulting viscosity increases.

However, as the addition of DAC also shows the same behavior of APA, there is no viscosity peak found, which means that as the shear rates increase, the viscosity decreases, suspension showed a shear thinning behavior, and there is no agglomeration as shear rate increases. The stabilization of DAC was assumed to have higher electrostatic stabilization compared with APA, as it was shown by the zeta potential result.

3.4. The Prediction of the Adsorbed Polymer. The prediction of the adsorbed dispersant agent into the 3Y-TZP surface was analyzed by the adsorption peak of FTIR. FTIR analysis was performed on APA, DAC, and 3Y-TZP before and after adsorption of $3.5 \mathrm{wt} . \%$, as seen in Figures 6(a) and 6(b), respectively. The assignments of the various bands and peaks made in this study are in reasonable agreement with those reported in the literature for similar functional groups [20-23]. The FTIR spectrum of APA shows strong absorption bands at 1,636 and $1,543 \mathrm{~cm}^{-1}$, indicating $-\mathrm{C}=\mathrm{O}$ stretching vibration for carboxylic acids. The bands at 1,443, $1,401,1,351$, and $1,319 \mathrm{~cm}^{-1}$ are due to the $\mathrm{C}-\mathrm{O}$ stretching vibration, and the broad peak at $3,208 \mathrm{~cm}^{-1}$ is attributed to the hydroxyl group $(\mathrm{O}-\mathrm{H})$ due to water. Meanwhile, the FTIR spectrum of $3 \mathrm{Y}$-TZP shows intense broad bands in the vicinity of $500 \mathrm{~cm}^{-1}$ and $1,636 \mathrm{~cm}^{-1}$, which are attributed to the stretching vibration of $\mathrm{m}-\mathrm{ZrO}_{2}$ and $\mathrm{t}-\mathrm{ZrO}_{2}$, as expected for $\mathrm{ZrO}_{2}$ samples. The spectrum showed peaks attributable to the $\mathrm{O}-\mathrm{H}$ group because the powder was in the liquid form. The adsorption of APA into the 3Y-TZP surface was investigated as shown in Figure 6(a), and new peaks were found at 1,563,1,551,1,534,1,508, 1,457, and $1,416 \mathrm{~cm}^{-1}$, indicating that $-\mathrm{C}=\mathrm{O}$ and $-\mathrm{C}-\mathrm{O}$ stretching vibrations were obtained from pure APA. On the other hand, as shown in Figure 5(b), suspensions with DAC as a dispersant agent also showed a shifted peak before and after the addition of a dispersant agent. The shift peak occurred at 1,585 and $1,396 \mathrm{~cm}^{-1}$, respectively, indicating $-\mathrm{C}=\mathrm{O}$ and $-\mathrm{C}-\mathrm{O}$ stretching vibrations. These findings proved that $\mathrm{APA}$ and $\mathrm{DAC}$ were adsorbed onto the surface of the $3 \mathrm{Y}$-TZP particles.

3.5. Zeta Potential Result. For the complete understanding of the stabilization of $3 \mathrm{Y}$-TZP with the addition of both dispersant agents, this was studied by the zeta potential to measure the electrostatic stabilization. Well-dispersed ceramic powders in suspensions could be obtained at high zeta potential absolute values by the addition of dispersant agents [13] (Figure 7).

Figure 7 shows that zero point charge (ZPC) of 3Y-TZP occurs at $\mathrm{pH}$ 9; however, as the dispersant agent was added, the IEP of the suspension shifts to a lower value as it is shown 


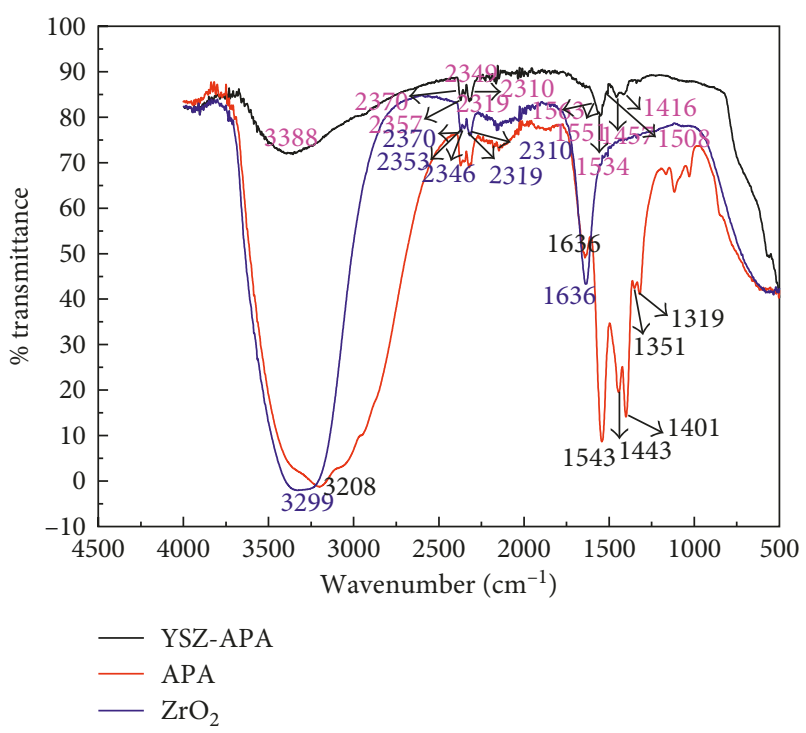

(a)

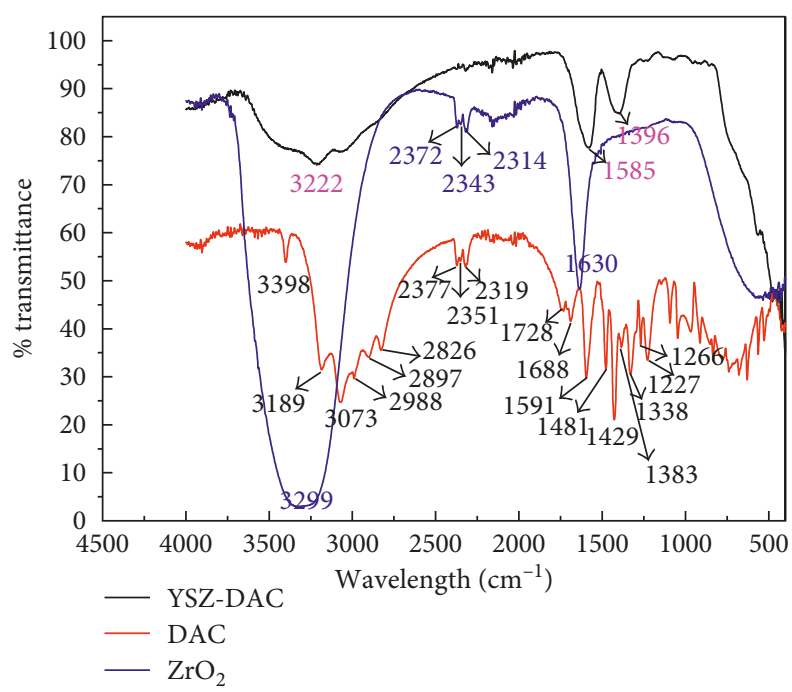

(b)

FIgURE 6: (a) The FTIR spectra of 3Y-TZP, APA, and 3Y-TZP-APA and (b) those of 3Y-TZP, DAC, and 3Y-TZP-DAC.

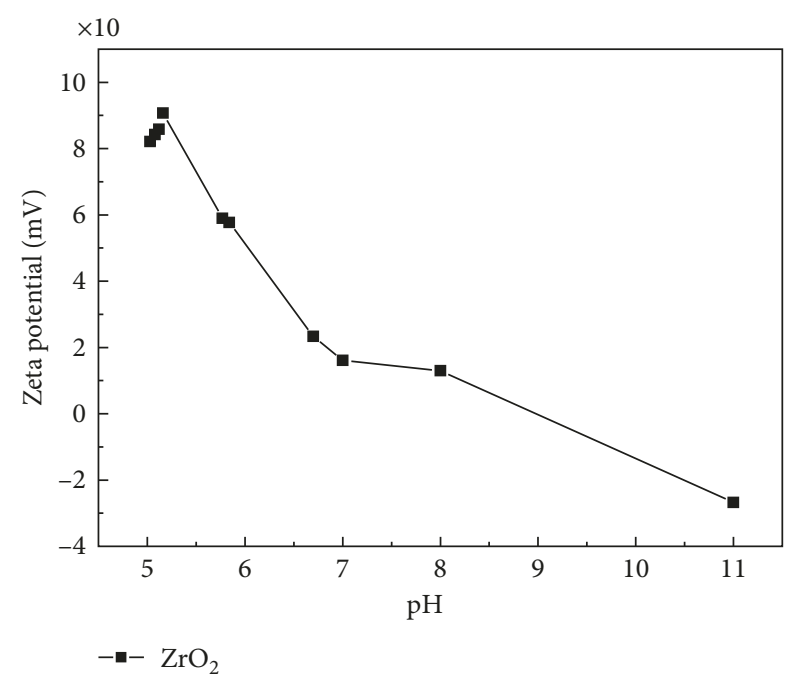

FIGURE 7: 3Y-TZP zeta potential of pure 3Y-TZP in water.

in Figure 8. The decrease in IEP is supposed to occur because both dispersant agents contain a carboxyl group that can produce a negative charge when dissolved in an aqueous solution, as shown in the following equation:

$$
\mathrm{RCOONH}_{4} \rightarrow \mathrm{RCOO}^{-}+\mathrm{NH}_{4}^{+}
$$

The negative charge of the carboxyl group is very easy to absorb into the positively charged zirconia, leading to the shift of IEP into a lower $\mathrm{pH}$ value. As shown in Figure 8(a), the addition of APA as a dispersant agent and the zeta potential of the powder surface do not change much from the initial $\mathrm{pH}$ value, except the strong acidic range and higher zeta potential cannot be accepted in a more basic condition. This means that the electrosteric stabilization also decreases as the $\mathrm{pH}$ increases, so we assumed that electrosteric stabilization can occur at $\mathrm{pH}$ below the ZPC.
This condition is also supported by a previous study that found that the loop and tail configuration of a polymer at a low $\mathrm{pH}$ will be dominant, while at higher $\mathrm{pH}$ values up to the ceramic's IEP, a train configuration of a stretched negatively charged polymer adsorbed onto positively charged surface sites is considered to exist.

At $\mathrm{pH}$ values above the IEP, electrostatic repulsions between the negative powder and negative polymer cause the linear polyelectrolyte to dangle into the solution, only adsorbing to the surface at a few minor regions with a positive surface charge $[9,24]$. Nevertheless, the addition of APA from all suspension concentrations found that $3.5 \mathrm{wt} \%$ obtained the highest zeta potential value in the $\mathrm{pH}$ range for all suspensions. It was found that the highest zeta potential was achieved at around $\mathrm{pH} 9$, where it could also support the yield of sedimentation; the $3.5 \mathrm{wt}$.\% suspension had the slowest sedimentation rate; and the $\mathrm{pH}$ of the dilute suspension was noted at 9.42 .

Similarly to APA, the addition of DAC shifted the IEP into the lower value. As it is shown in Figure 8(b), the addition of DAC as a nonpolymeric dispersant showed higher zeta potential values which occurred at lower $\mathrm{pH}(\mathrm{pH} 7)$. This result also supported that the sedimentation result at $3.5 \mathrm{wt} . \%$ DAC concentration was noted to have $\mathrm{pH}$ 6.82. At high concentrations, addition over $2.5 \mathrm{wt}$. $\%$ showed a large zeta potential value in the negative charge for the $\mathrm{pH}$ range 6-7, but a large zeta potential value in the positive charge was observed for $1.5 \mathrm{wt}$ \% addition of dispersant agents. Adding a strong acid to the higher concentration of DAC suspensions will give DAC the ability to bond with hydrogen ions and form a weak acid and thus decrease the solution's acidity but not for a low concentration of DAC because as the strong acid increases, the solution's acidity will keep increasing so that solutions will have a high zeta potential value in the positive charge for $1.5 \mathrm{wt} \%$ addition. In accordance with the zeta potential result, we can understand 


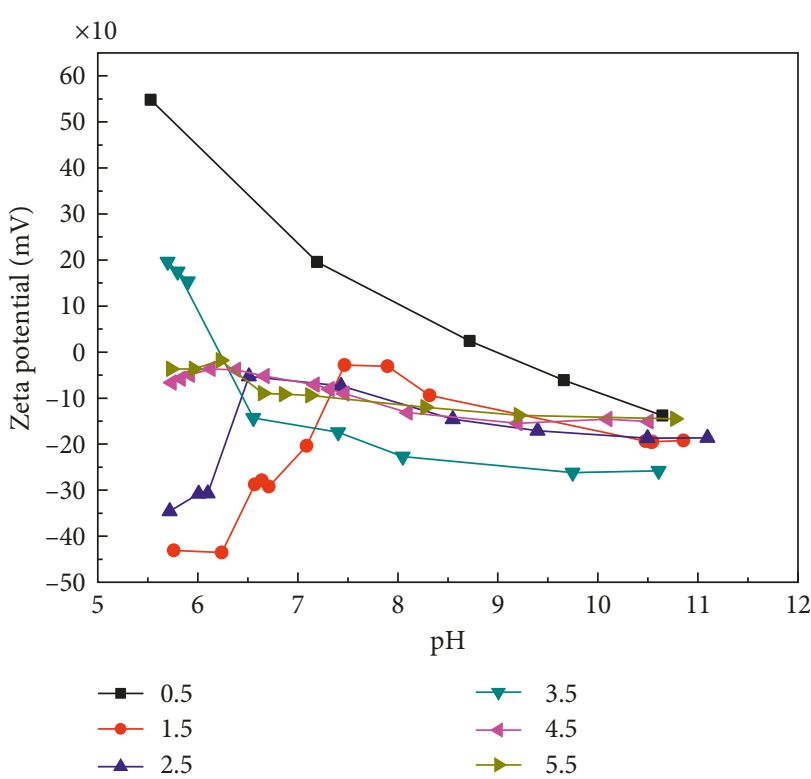

(a)

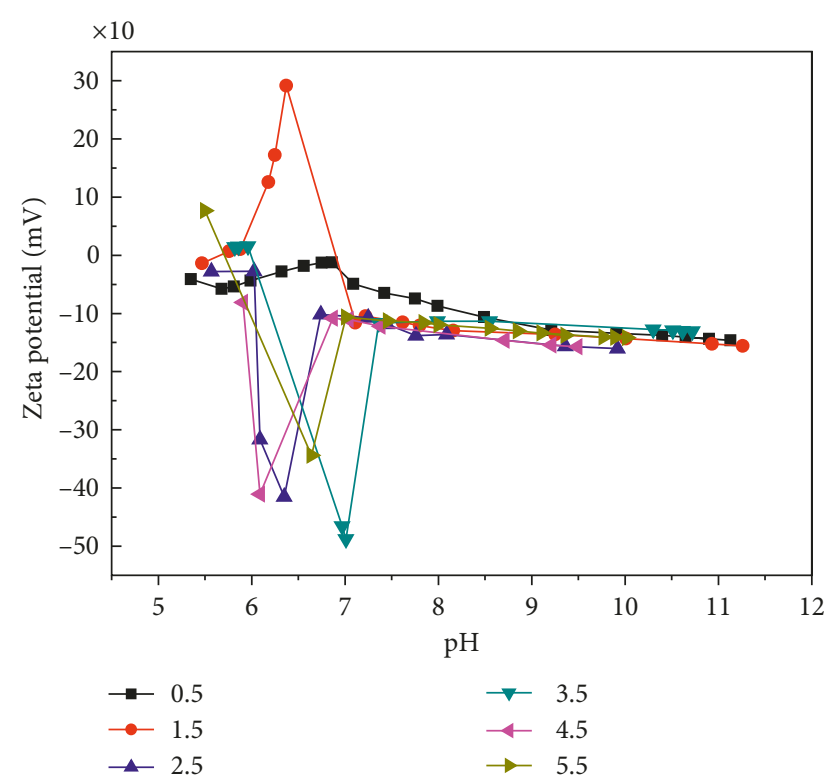

(b)

FIgURE 8: 3Y-TZP zeta potential with the addition of 0.5-5.5 wt.\% of dispersant agents: (a) APA addition and (b) DAC addition.

that electrostatic stabilization occurred more with the addition of DAC compared to the addition of APA. The reason could be due to the $\mathrm{pH}$ of the suspension. Since both of dispersants contain the carboxyl group, it is very easy to adsorb on the positive side of zirconia, and thus, it will make the particle negatively charged and reduce the IEP to the lower value. At low $\mathrm{pH}$, adsorption of the adsorbate will occur by a flat adsorbtion conformation and thus will make the particle adhere to each other and result in higher zeta potential as shown in the DAC case. But as $\mathrm{pH}$ of the suspension is higher, the adsorbate will dangle into solution, thus decreasing the zeta potential value as it was found in the APA case.

\section{Conclusions}

Both APA and DAC were found to be effective dispersant agents for 3Y-TZP; the smallest particle size, the small tendency towards sedimentation, and the higher zeta potential became critical values for understanding the stabilization of the suspension, where these values can be achieved with the addition of both APA and DAC at $3.5 \mathrm{wt} . \%$.

APA is a kind of the polyelectrolyte that becomes a good candidate for stabilizing the 3Y-TZP suspension via electrostatic and steric stabilization. Nevertheless, steric stabilization is dominating because of the high $\mathrm{pH}$ value of APA suspensions. Differing from APA, DAC which also contain carboxyl group but has lower $\mathrm{pH}$ than APA will stabilize the suspension via electrosteric stabilization.

\section{Conflicts of Interest}

The authors declare that they have no conflicts of interest.

\section{Acknowledgments}

This work was supported by Kyungnam University Foundation Grant 2016.

\section{References}

[1] T. Masaki, "Mechanical properties of toughened $\mathrm{ZrO}_{2} \mathrm{Y}_{2} \mathrm{O}_{3}$ ceramics," Journal of the American Ceramic Society, vol. 69, no. 8, pp. 638-640, 1986.

[2] D. L. Porter and A. H. Heuer, "Mechanism of toughening partially stabilized zirconia (PSZ)," Journal of the American Ceramic Society, vol. 60, pp. 183-184, 1977.

[3] M. Aizawa, K. Itatani, F. S. Howell, A. Kishioka, and M. Kinoshita, "Formation of porous calcium phosphate films on partially stabilized zirconia substrates by the spraypyrolysis technique," Journal of Materials Science, vol. 30, no. 19, pp. 4936-4942, 1995.

[4] D. W. Richerson, Modern Ceramics Engineering Properties, Processing and Use in Design, Marcel Dekker, New York, NY, USA, 2nd edition, 1992.

[5] T. Fengqiu, H. Xiaoxian, Z. Yufeng, and G. Jingkun, "Effect of dispersant agent on the surface chemical properties of nanozirconia suspensions," Ceramics International, vol. 26, no. 1, pp. 93-97, 2000.

[6] J. Davies and J. G. P Binner, "The role of polyacrylate in dispersing concentrated alumina suspensions," Journal of the European Ceramic Society, vol. 20, no. 10, pp. 1539-1553, 1999.

[7] B. V. Velamakanni, J. C. Chang, F. F. Lange, and D. S. Pearson, "A new method for efficient colloidal particle packing via modulation of repulsive lubricating hydration forces," Langmuir, vol. 6, no. 7, pp. 1323-1325, 1990.

[8] R. Greenwood and K. Kendall, "Selection of suitable dispersants for aqueous suspensions of zirconia and titania powders using acoustophoresis," Journal of the European Ceramic Society, vol. 19, no. 4, pp. 479-488, 1999. 
[9] J. Cesarano and I. A. Aksay, "Processing of highly concentrated aqueous $\alpha$-alumina suspensions stabilizes with polyelectrolytes," Journal of the American Ceramic Society, vol. 71, no. 12, pp. 1062-1067, 1998.

[10] M. Hashiba, H. Okamoto, Y. Nurishi, and K. Hiramasu, "Dispersion of $\mathrm{ZrO}_{2}$ particles in aqueous suspensions by ammonium polyacrylate," Journal of Materials Science, vol. 24 , no. 3, pp. 219-224, 1989.

[11] P. C. Hidber, T. J. Graule, and L. J. Gauckler, "Influence of the dispersant structure on properties of electrostatically stabilized aqueous alumina suspension," Journal of the European Ceramic Society, vol. 17, no. 2-3, pp. 239-249, 1997.

[12] Pradip, R. S. Premachandran, and S. G. Malghan, "Electrokinetic behavior and dispersion characteristics of ceramic powders with cationic and anionic polyelectrolytes," Bulletin of Materials Science, vol. 17, no. 6, pp. 911-920, 1994.

[13] Z. Xie, J. Ma, Q. Xu, Y. Huang, and Y.-B. Cheng, "Effects of dispersants and soluble counter-ions on aqueous dispersibility of nano-sized zirconia powder," Ceramics International, vol. 30, no. 2, pp. 219-224, 2004.

[14] A. E. Regazzoni, M. A. Blesa, and A. J. G. Maroto, "Interfacial properties of zirconium dioxide and magnetite in water," Journal of Colloid and Interface Science, vol. 91, no. 2, pp. 560-570, 1983.

[15] S. Kim, K. Hyun, J. Y. Moon, C. Clasen, and K. H. Ahn, "Depletion stabilization in nanoparticle-polymer suspensions: multi length-scale analysis of microstructure," Langmuir, vol. 31, no. 6, pp. 1892-1900, 2015.

[16] I. Ganesh, G. Sundararajan, S. M. Olhero, P. M. C. Torres, and J. M. F. Ferreira, "A novel colloidal processing route to alumina ceramics," Ceramics International, vol. 36, no. 4, pp. 1357-1364, 2010.

[17] M. Acosta, V. L. Wiesner, C. J. Martines, R. W. Trice, and J. P. Youngblood, "Effect of polyvinylpyrrolidone additions on the rheology of aqueous, highly loaded alumina suspensions," Journal of the American Ceramic Society, vol. 96, no. 5, pp. 1372-1382, 2013.

[18] J. S. Chong, E. B. Christiansen, and A. D. Baer, "Rheology of concentrated suspensions," Journal of Applied Polymer Science, vol. 15, pp. 2007-2021, 1971.

[19] B. Lennart, Colloidal Processing, Chapter 9, Institute for Surface Chemistry, Stockholm, Sweden, 2001.

[20] D. Santhiya, S. Subramanian, K. A. Natarajan, and S. G. Malghan, "Surface chemical studies on the competitive adsorption of poly(acrylic acid) and poly(vinyl alcohol) onto alumina," Journal of Colloid and Interface Science, vol. 216, no. 1, pp. 143-153, 1999.

[21] Q. Ran, S. Wu, and J. Shen, "Effects of poly(acrylic acid) on rheological and dispersion properties of aqueous $\mathrm{TiO}_{2}$ suspensions," Polymer-Plastics Technology and Engineering, vol. 46, no. 11, pp. 1117-1120, 2007.

[22] S. Liufu, H. Xiao, and Y. Li, "Adsorption of poly(acrylic acid) onto the surface of titanium dioxide and the colloidal stability of aqueous suspension," Journal of Colloid and Interface Science, vol. 281, no. 1, pp. 155-163, 2005.

[23] S. H. Othman, S. A. Rashid, T. I. M. Ghazy, and N. Abdullah, "Dispersion and stabilization of photocatalytic $\mathrm{TiO}_{2}$ nanoparticles in aqueous suspension for coatings applications," Journal of Nanomaterials, vol. 2012, Article ID 718214, 10 pages, 2012.

[24] J. Cesarano, I. A. Aksay, and A. Bleier, "Stability of aqueous $\mathrm{Al}_{2} \mathrm{O}_{3}$ suspensions with poly(methacrylic acid) polyelectrolyte," Journal of the American Ceramic Society, vol. 71, no. 4, pp. 250-255, 1988. 


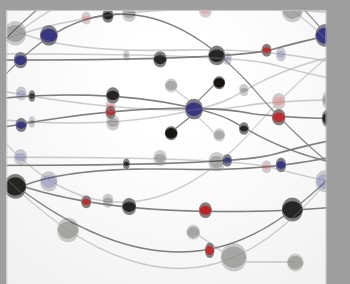

The Scientific World Journal
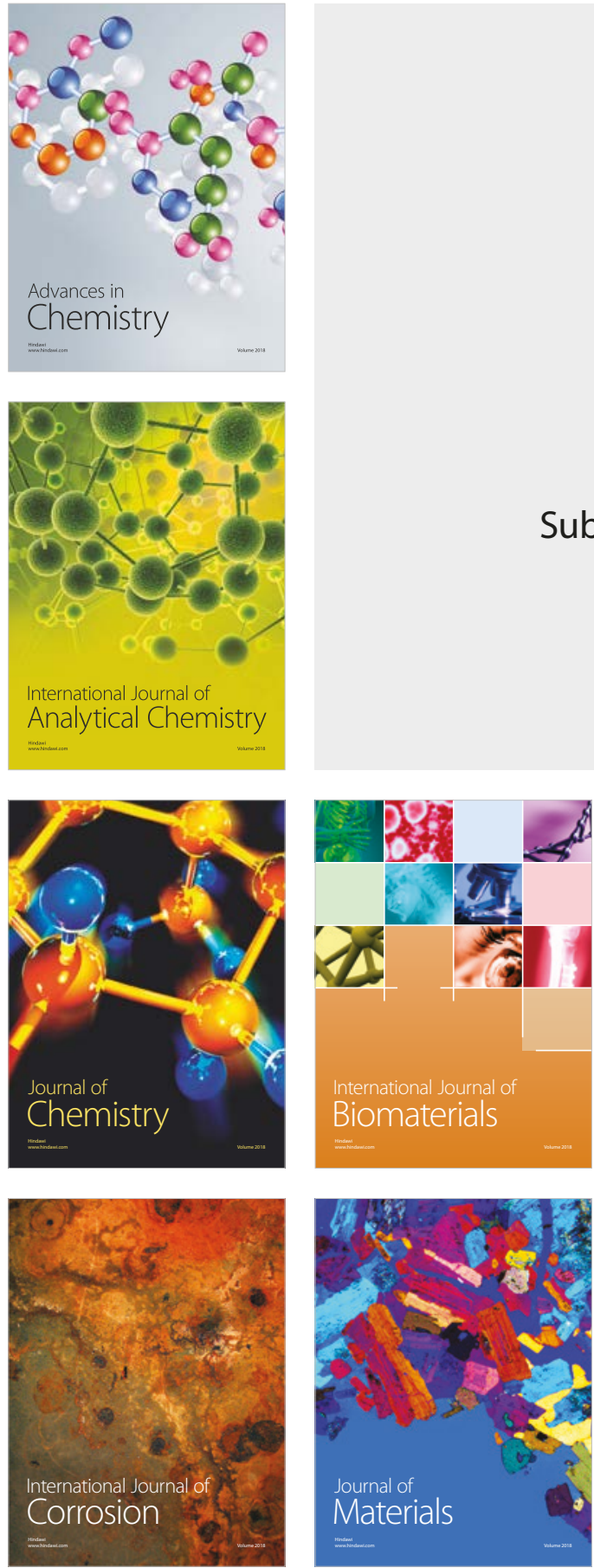

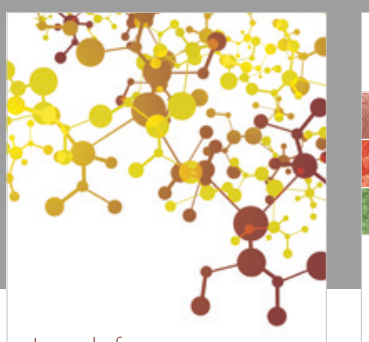

Journal of

Applied Chemistry
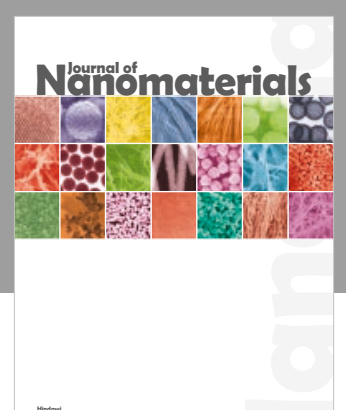

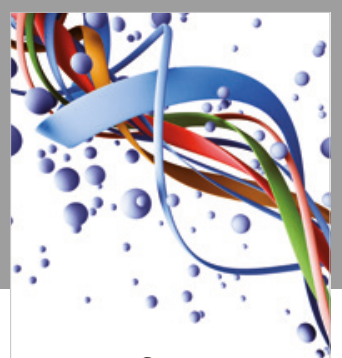

Scientifica

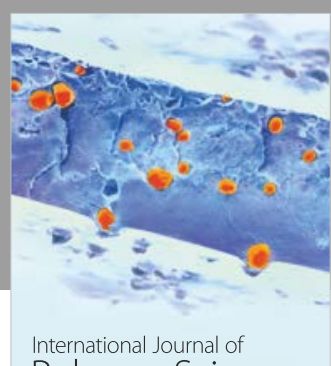

Polymer Science

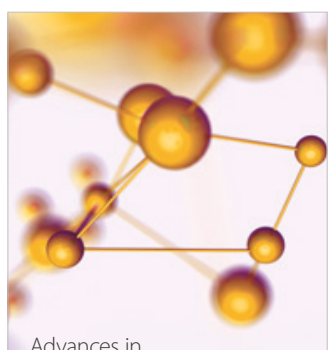

Physical Chemistry
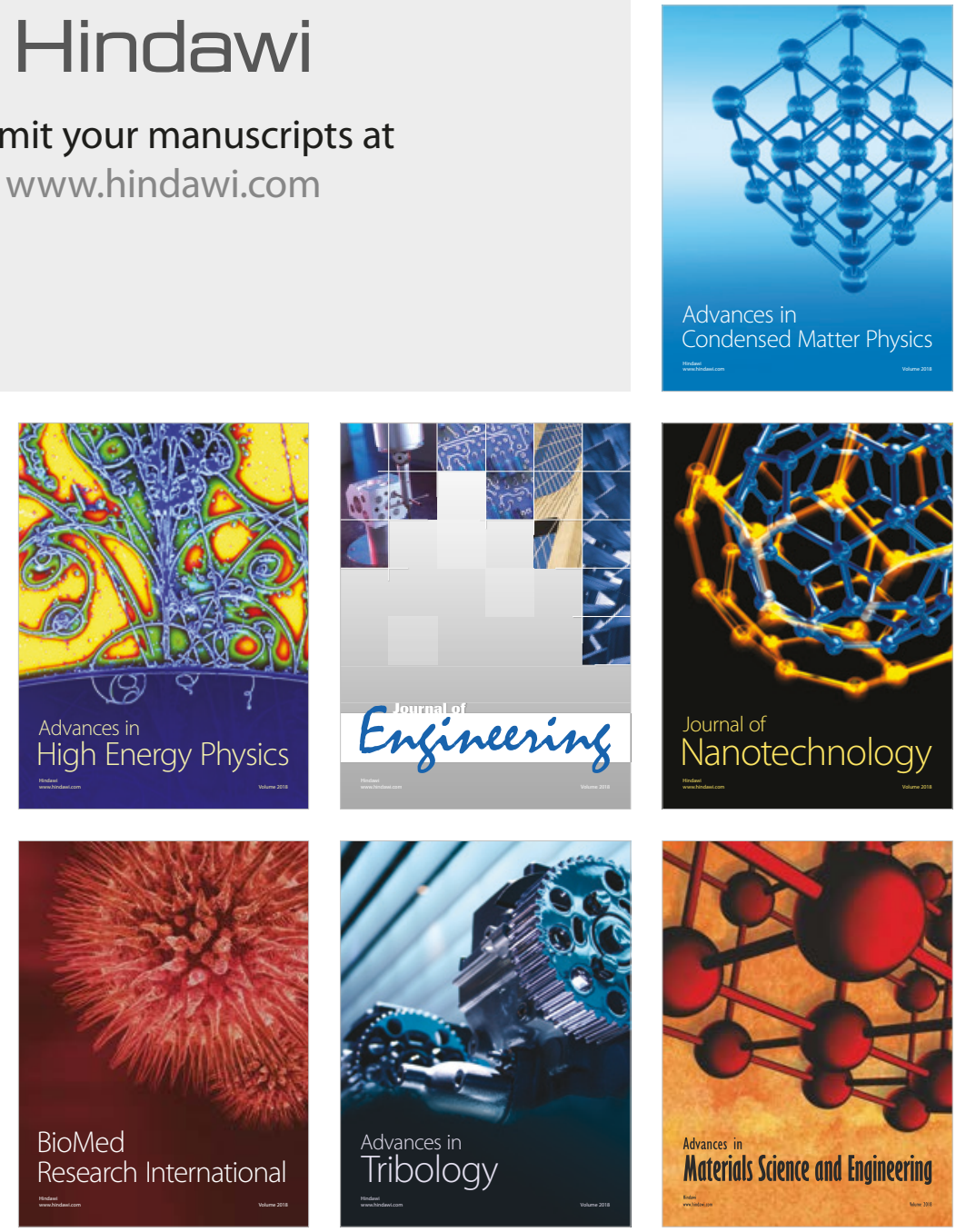